\title{
Experimental demonstration of a global dispersion-free steering correction at the new linac test facility at SLAC
}

\author{
A. Latina, J. Pfingstner, and D. Schulte \\ European Organization for Nuclear Research (CERN), Geneva, Switzerland \\ E. Adli \\ Department of Physics, University of Oslo, 0316 Oslo, Norway \\ F. J. Decker and N. Lipkowitz \\ SLAC National Accelerator Laboratory, Menlo Park, California 94025, USA
}

(Received 10 October 2013; published 11 April 2014; corrected 1 May 2014)

\begin{abstract}
The performance of future linear colliders will depend critically on beam-based alignment and feedback systems. In ILC and CLIC it is planned to perform dispersion-free steering in the main linacs. To this end the beams are accelerated with different gradients to evaluate the dispersion. The steering is performed by minimizing the average offset of the different beams in the beam position monitors and, at the same time, the difference between the beam trajectories. The experimental verification of the dispersion-free steering algorithm is essential to prove its effectiveness and to prepare the commissioning of such machines. The algorithm should take an orbit measurement at every cycle (train to train), estimate the correction from this information, and, from the system response matrices, apply the correction. We have successfully tested dispersion-free steering at FACET, including an adaptive system-identification algorithm, where the system response matrix is measured dynamically and automatically.
\end{abstract}

DOI: 10.1103/PhysRevSTAB.17.042803

29.20.Ej, 29.27.Eg

\section{INTRODUCTION}

The proposed high-luminosity, electron-positron linear colliders CLIC [1] and ILC [2] require normalized vertical emittances of the order of $10 \mathrm{~nm}$ at the interaction point in order to reach the target luminosities of $10^{34} / \mathrm{cm}^{2} / \mathrm{s}$. Two of the main sources of emittance growth are: the emittance dilution due to spurious dispersion in the quadrupoles, which scales as the absolute misalignment of the beam position monitors (BPMs) squared, and the emittance dilution due to transverse wake fields which scales as the square of the accelerator structure offset [3]. For instance, the main linacs of the CLIC machine at $3 \mathrm{TeV}$ center of mass energy are $21 \mathrm{~km}$ long and contain about 2000 quadrupoles and BPMs [1]. Due to the sheer amount of components, local BBA alignment techniques are impractical and excessively time consuming. Instead, in order to align such linacs in a robust and more practical way, global correction algorithms have been studied intensively during the last decades [4-7]. For both CLIC and ILC global dispersion-free correction algorithms [4]

*andrea.latina@cern.ch

Published by the American Physical Society under the terms of the Creative Commons Attribution 3.0 License. Further distribution of this work must maintain attribution to the author $(s)$ and the published article's title, journal citation, and DOI. have been proposed and foreseen as part of the linac tuning procedures $[1,2]$.

In this paper we study a variant of dispersion-free correction, which is based solely on a model of the linear response of each BPM to a corrector kick. We summarize such an information in the system response matrix, $\mathbf{R}$, defined as

$$
R_{i j}=\frac{\partial x_{i}}{\partial \theta_{j}}
$$

where $x_{i}$ is the position of BPM $i$ and $\theta_{j}$ the set-point of corrector $j$. To first order, $R_{i j}$ is identical to the element $M_{12}$ of the linear transfer matrix $M$ from the $j$ th corrector to the $i$ th BPM. The response matrix is calculated using orbit differences and, to first order, is independent of the absolute beam trajectory. This is also true for the effect of the dipole mode of the transverse wake, which is linear in the transverse coordinates [8]. The response matrix can in principle be extracted from a computer model of the machine. The accuracy of the response matrix then depends on the quality of the model. In this paper we study an alternative approach where this matrix is inferred from repeated measurements on the machine. This approach has the advantage that all linear contributions to the response matrix are correctly included. We have developed a system identification algorithm that automatically combines several measurements [9]. 
The knowledge of the response matrix allows us to apply beam-based alignment techniques. Among these techniques, dispersion-free steering (DFS) is recognized as one of the most effective in preserving the low emittances of future linear colliders. DFS aims at correcting orbit and dispersion simultaneously, effectively overcoming systematic errors due to BPM offsets.

For the feasibility studies of linear colliders, an experimental proof of principle of the linac tuning algorithms, including DFS, is highly anticipated as most of emittance preservation studies rely solely on simulations. Early experimental tests of beam-based alignment were performed at the Stanford Linear Collider (SLC), as reviewed in Ref. [10], however did not give fully conclusive results and did not demonstrate emittance reduction [11]. One reason given for the limited performance was reported to be a potential mismatch between the model used to compute the correction, entirely based on an ideal computer lattice and the real machine.

Other experimental studies of beam-based alignment were later performed using a variant of dispersion-free steering, called "two-beam" dispersion-free steering, both at the SLC [10] and at the circular collider LEP [12]. The two-beam dispersion correction consisted of applying DFS measuring the dispersion as the difference between the orbits of the electron and positron beams. Two-beam DFS was applied with great success at LEP: the vertical dispersion was reduced to the expected design minimum, with a significant impact on the vertical emittance and on the luminosity. The same technique was less successful at the SLC, where it led to a vertical emittance at the end of the linac as large as 2-3 times the value at injection [13]. The difference between the two-beam DFS performance at the SLC and at LEP can be understood if one considers how different the machine knowledge is when operating with a ring or with a linac. In the case of a ring repeated measurements on the closed orbit can be performed to precisely measure the machine optical properties prior to compute a correction, whereas the bunches travel a linac only once. Mismatches between the ideal and the real optics are thus much harder to identify in a linac. Furthermore, neither in ILC nor in CLIC will the electrons and the positrons share the same beam-pipe, rendering impossible such an efficient "two-beam" dispersion measurement. It is worth recalling that the sensitivity of DFS to energy errors scales with $1 / \Delta E$ (the effective energy change used to measure the dispersion). Using electrons and positrons gives an effective energy difference of $200 \%$, which greatly improves the performance of DFS. In a linac with only electrons, or only positrons, the feasible energy difference that can typically be achieved amounts to about $5-10 \%$ of the beam energy, posing an additional challenge that emphasises the relevance of the study here presented.

With the availably of a new linac test facility, the FACET test-facility at SLAC [14], which makes use of the first $2 \mathrm{~km}$ of the Stanford Linear Collider, tuning algorithms for linear colliders can again be tested experimentally. In this paper we report on the first experimental proof of principle of emittance reduction in a long linac by applying dispersion-free correction using automatic system-identification algorithms.

The paper is organized as follows: First, we describe the system identification approach used to construct the response matrix. Then, we present the dispersion-free correction technique, and verify the combined effect of the system identification and dispersion-free correction by PLACET [15] simulations of a model of the FACET linac. Finally, we report on experimental results from the FACET linac where system identification and dispersion-free correction, tested on a computer model, were applied in an identical manner to the real machine.

\section{SYSTEM IDENTIFICATION}

The orbit response of an accelerator to its correctors provides important information to many beam-based methods used during the operation of a modern particle accelerator. Examples include beam-based alignment, orbit feedback systems, diagnosis and error detection tools. The orbit response is a matrix usually measured by exciting each corrector one after the other and recording the excited trajectory. The achievable accuracy of this method is limited by the maximal excitation strengths and by the resolution of the BPMs. In Ref. [9] we have successfully studied the possibility of using many small excitations over time to gradually improve the system knowledge with the help of system identification algorithms that optimally reduce the effects of the measurement noise.

System identification is the process of estimating unknown parameters of a system with known structure by measurements of its response. This problem is addressed by exciting the measured system with a given set of actuators and using the system response to estimate the unknown parameters. A schematic of the algorithm is shown in Fig. 1. An important requirement for system identification is that the excitation is broad enough to sample the whole parameter space of the system to be identified. In the case of particle accelerators the maximal allowable beam excursion can become a limiting factor. For this reason, the excitation has to be chosen such that every beam excursion $x_{i k}$ in the $i$ th BPM due to an excitation of the $k$ th corrector stays below a given maximal excursion $\left|x_{i k}\right|<|\hat{x}|$. The beam excursion $x_{i k}$ can be written as

$$
x_{i k}=\sqrt{\beta_{i} \beta_{k}} \sin \left(\phi_{i}-\phi_{k}\right) \sqrt{\frac{E_{k}}{E_{i}}} \theta_{k},
$$

where $\beta$ and $\phi$ represent the $\beta$-function and the phase advance at a certain position, $E$ the beam energy, and $\theta_{k}$ is the kick applied to the beam by the corrector. This formula 


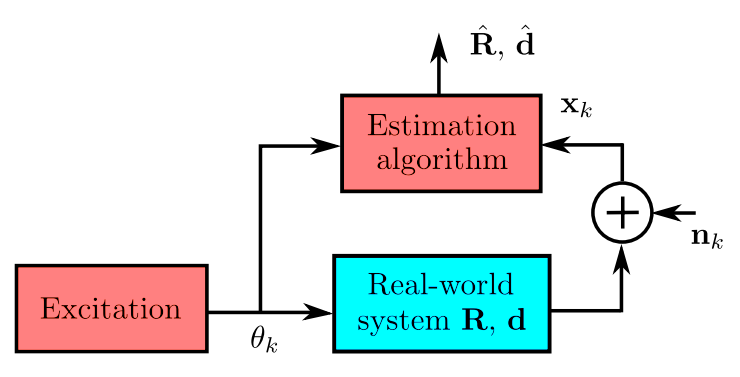

FIG. 1. General system identification scheme with the corrector set points $\theta_{k}$, the BPM measurements $x_{k}$, and the BPM noise $n_{k}$. In the case of system identification for orbit response matrices the system parameters to be identified are the orbit response matrix $R$ and the reference orbit $d$, where the hat index is used to distinguish between estimated and real parameters.

suggests that BPMs and correctors located at large $\beta$ functions are favorable, and allows us to estimate the required kick to excite an arbitrary oscillation. At FACET we chose to use $1 \mathrm{~mm}$ large excitations in the horizontal direction, and $0.5 \mathrm{~mm}$ in the vertical one. We furthermore selected a subset of BPMs and correctors with large beta functions, according to Eq. (1), to maximize the signal-tonoise ratio of our measurements.

To estimate the response matrix, different algorithms can be found in the literature, see for instance $[16,17]$. In our study we focused only on the algorithms that can be formulated in a recursive form (very well suited for online calculation), and ultimately chose the recursive least squares (RLS) algorithm: a recursive variant of the wellknown least squares (LS) solution. Figure 1 depicts the block diagram of this algorithm: the correctors are indicated with $u_{k}$, the BPMs with $y_{k}$, and the BPM noise with $n_{k}$. The response matrix to be identified is $R$, and $d$ is the reference orbit.

\section{BEAM-BASED ALIGNMENT}

Beam-based techniques use beam measurements to infer beam properties such as orbit and dispersion, and to improve the machine performance based on this information. Such techniques include for example: quadrupoleshunting, beam-based alignment, energy monitoring, feedback loops, etc. Among the alignment algorithms, "one-to-one" $(1: 1)$ is a correction technique that tries to steer the beam to its nominal trajectory by minimizing the reading of each BPM using appropriate corrector strengths. This technique is useful to ensure the beam travels through the machine without hitting the walls, but in general is not sufficient to preserve nanometer-scale emittances. The reason is that $1: 1$ correction does not take into account the systematic errors introduced by misaligned BPMs. During the last decades improved algorithms have been developed to overcome this limitation: one example is DFS, first presented in Ref. [4]. DFS is a variant of $1: 1$ correction that attempts simultaneously to steer the beam to its nominal orbit and to correct the beam dispersion, by minimizing the following merit function:

$$
\chi^{2}=\sum_{\text {bpms }} x_{i}^{2}+\omega^{2} \sum_{\text {bpms }}\left(x_{i}^{\prime}-x_{i}\right)^{2}+\kappa^{2} \sum_{\text {corrs }} \theta_{j}^{2},
$$

where $\boldsymbol{\theta}$ is the vector of unknowns, $\theta_{j}$ (the corrector strengths); and the vectors $\mathbf{x}, \mathbf{x}^{\prime}$ are, respectively, the BPM readings for the nominal and the dispersive beams, $x_{i}$ and $x_{i}^{\prime}$. The parameters $\kappa$ and $\omega$ are free and need to be tuned to achieve best performance: $\omega$ is a weighting factor balancing between orbit and dispersion correction, $\kappa$ can be used to limit the amplitude of the corrections (increasing $\kappa$ is equivalent to cutting the least significant singular values of the system matrix, larger values of $\kappa$ effectively cut more singular values). The factor $\kappa$ is generally chosen empirically, but the weighting factor $\omega$ can be estimated using the formula:

$$
\omega^{2}=\frac{\sigma_{\mathrm{bpmoffset}}^{2}+\sigma_{\mathrm{bpm} \text { precision }}^{2}}{2 \sigma_{\mathrm{bpm} \text { precision }}^{2}} .
$$

A careful choice of the free parameters is crucial to achieve optimal performance. The least squares solution of Eq. (2) with respect to the correctors can be written as the following system of equations,

$$
\left[\begin{array}{c}
\mathbf{x} \\
\omega \cdot\left(\mathbf{x}-\mathbf{x}^{\prime}\right) \\
\mathbf{0}
\end{array}\right]=\left[\begin{array}{c}
\mathbf{R} \\
\omega \cdot\left(\mathbf{R}-\mathbf{R}^{\prime}\right) \\
\kappa \cdot \mathbf{I}
\end{array}\right] \boldsymbol{\theta},
$$

where $\mathbf{R}$ and $\mathbf{R}^{\prime}$ are the response matrices of the nominal and the test beams used to quantify the dispersion; $\mathbf{I}$ is the identity matrix.

Dispersion-free steering requires a test-beam that follows a dispersive trajectory to measure the dispersion. A few techniques are routinely used to measure the dispersion: one of them consists of creating an energy difference upstream the section to be corrected and then measuring the orbit deviation in the BPMs. We identified two possible methods for creating such an energy difference at FACET: (i) moving the klystrons phase shifters to modify the energy gain in some sectors; (ii) changing the klystron amplitudes (or switching off some klystrons). We adopted the first method, as it seems the most practical to implement. If positrons will be available in the future, BPM readings from both electron and positron trains could be combined to obtain a dispersion measurement.

\section{SIMULATION OF THE EXPERIMENT}

A simulation of the entire experiment has been carried out to evaluate the performance of dispersion-free steering and system-identification algorithms at FACET, using the tracking code PLACET. In simulation, we chose to use a BPM resolution of $5 \mu \mathrm{m}$ : this is compatible with the BPM 
TABLE I. Misalignment and BPM precision values used for in the FACET linac simulation.

\begin{tabular}{lc}
\hline \hline Symbol & Value, RMS \\
\hline$\sigma_{\text {quadrupole offset }}$ & $100 \mu \mathrm{m}$ \\
$\sigma_{\text {bpm offset }}$ & $100 \mu \mathrm{m}$ \\
$\sigma_{\text {bpm precision }}$ & $5 \mu \mathrm{m}$ \\
\hline \hline
\end{tabular}

TABLE II. Relevant beam parameters at the injection of the FACET linac.

\begin{tabular}{lc}
\hline \hline Symbol & Value \\
\hline$\gamma \epsilon_{x}$ & $30 \mu \mathrm{m} \cdot \mathrm{rad}$ \\
$\gamma \epsilon_{y}$ & $2.5 \mu \mathrm{m} \cdot \mathrm{rad}$ \\
$\sigma_{z}$ & $1 \mathrm{~mm}$ \\
$\sigma_{E}$ & $1 \%$ \\
$q$ & $1.6 \mathrm{nC}$ \\
$E_{0}$ & $1.19 \mathrm{GeV}$ \\
\hline \hline
\end{tabular}

resolution achieved at FACET, as it is explained in the following sections. A summary of the relevant parameters of the simulation is presented in Tables I and II.

In the first part of our simulated experiment, the system identification algorithm has been applied over the linac in order to evaluate the measurement of the system response (that is, we did not use the theoretical response matrix). The convergence parameter we used to quantify how well the system-identification algorithm has learned the response matrix, $\xi$, is the relative "Frobenius" distance [the Frobenius distance is defined as : $\|R\|_{\mathrm{F}}=\sqrt{\operatorname{tr}\left(R R^{T}\right)}$ ] between the estimated and the ideal response matrices: $\xi=\left\|R_{\text {measured }}-R_{\text {ideal }}\right\|_{\mathrm{F}} /\left\|R_{\text {ideal }}\right\|_{\mathrm{F}}$.

The result of the simulations, showing the convergence for different values of the permitted excitation, are shown in Fig. 2. In this plot the black line, i.e., the line manifesting the quickest convergence, corresponds to the realistic case of $1 \mathrm{~mm}$ orbit oscillation like we have used during the experiment.

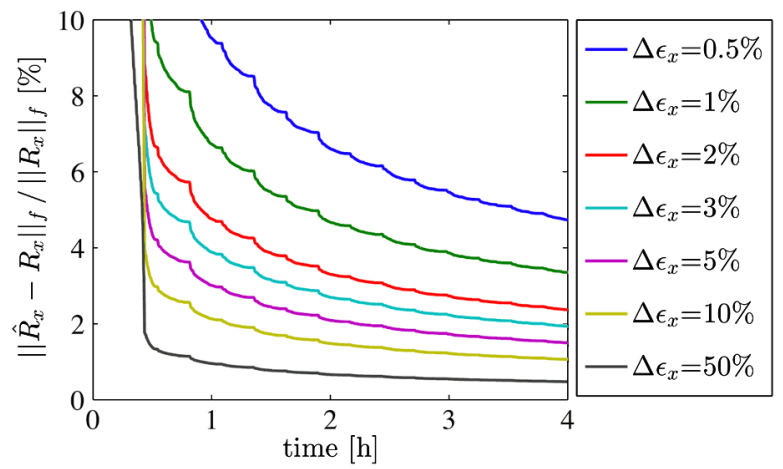

FIG. 2. Convergence of the system identification algorithm. A relative error of $1 \%$ is considered sufficient to perform BBA. The result is the average of 100 random seeds.
To apply DFS, we measured the dispersion by offsetting by 5 deg the phase of a sub-booster upstream the part of linac we applied the correction in. A careful optimization of the DFS free parameters resulted in $\kappa=1[\mu \mathrm{m} / \mathrm{kV}]$, $\omega=14$. The results of the simulations are shown in Fig. 3. It must be emphasized that we do not expect to obtain a perfect match between simulation and experimental measurements. The final result of the correction algorithms depends on several parameters that can significantly differ between the computer-simulated and the real experiment, for example: the focusing properties of the lattice, the choice of BPMs and correctors used during correction, how the energy difference is created to measure the dispersion, the input bunch length, the input bunch population, etc. However, since we reconstruct the machine response in both cases, these differences do not affect the performance of the correction as such. This performance can be evaluated in simulation, where it is shown that applying DFS reduces the emittance growth more effectively than a simple orbit-based correction. It must be noted that a residual emittance growth is still observable.

A further step we took to assess the performance of SYSID + DFS was to simulate the impact of an imperfect model knowledge on DFS itself; that is, apply DFS using response matrices spoiled by BPM random noise, instead of the ideal, theoretical, matrices. At this purpose we averaged the emittance growth of 1000 different random seeds, not just 100 like we did previously, because in this case the numerical sampling of the system must not just take into account the randomness of the misalignments, but also the arbitrariness of the response matrix; in fact each seed is subjected to a different set of misalignments and a different imperfect response matrix. Figure 4 shows the impact of a $1 \%$ error and $5 \%$ error on the performance on DFS.

An analysis of the performance of DFS has been carried out to achieve full understanding of the system in study.



FIG. 3. Emittance growth along the vertical axis before and after dispersion-free steering. The result is the average of 100 random seeds of misalignment. 


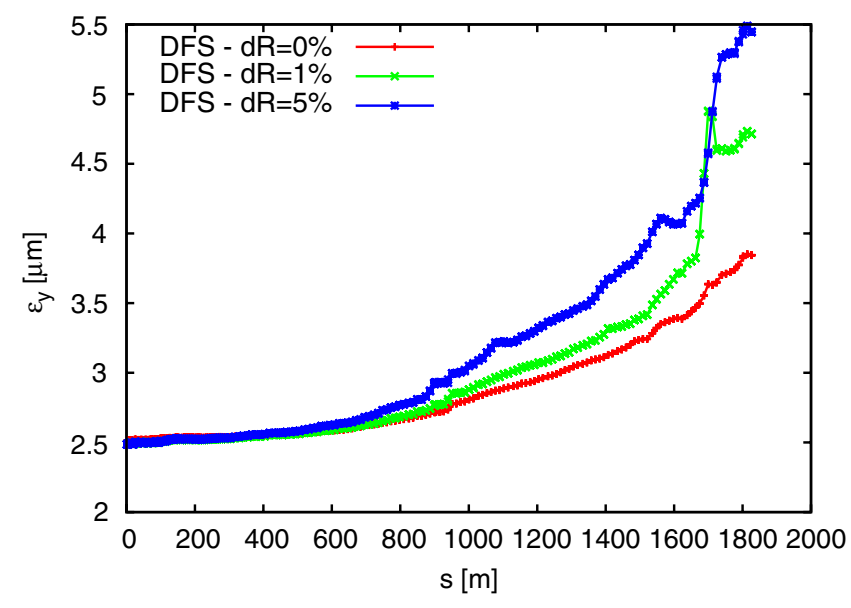

FIG. 4. Emittance growth after dispersion-free steering with imperfect model, compared to the case with perfect mode. The results are the average of 1000 random seeds.

DFS has been applied to simulated machines affected by only one single source of imperfection at the time; four cases have been studied: only misaligned quadrupoles, only misaligned cavities, only misaligned BPM with perfect resolution, and perfectly aligned BPMs only affected by measurement noise. Each single case has been computed for different values of the parameter $\omega$, effectively showing how the performance of 1:1 (small values of $\omega$ ) evolves toward more effective dispersion corrections (large values of $\omega$ ). Figure 5 shows the result of this simulation. The plot shows that the dominating contributions to emittance dilution is due to structure misalignments, which demonstrates the strong effect of the wakefields in the FACET linac.

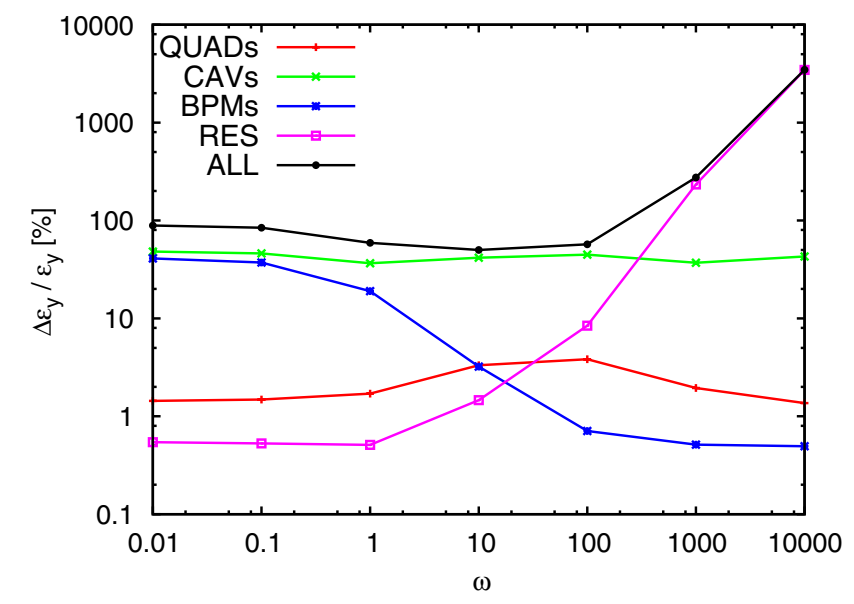

FIG. 5. Emittance growth after dispersion-free steering for each individual source of imperfection, as a function of the parameter $\omega$. Each point is the average of 100 random seeds. The dominating contribution to emittance dilution is due to structure misalignments, which demonstrates the strong effect of the wakefields on the FACET linac.

\section{EXPERIMENTAL SETUP}

As an experimental test bed we targeted a $500 \mathrm{~m}$ section of the SLAC FACET linac, starting $200 \mathrm{~m}$ downstream of the injection into the linac and ending $700 \mathrm{~m}$ downstream. The bunch length in this part of the linac was about $1 \mathrm{~mm}$. The bunch charge was $1.6 \mathrm{nC}$ and the energy at the starting point of the correction was $4 \mathrm{GeV}$. We denote as the "golden orbit" the initial electron orbit as steered manually by the FACET operators, before our correction.

Before dispersion correction an initial system identification for both the nominal orbit, $\mathbf{R}$, and the dispersive orbit, $\mathbf{R}^{\prime}$ is required. In order to achieve an effective BPM resolution of less than $5 \mu \mathrm{m}$, as shown to be required by simulation, 100 BPM samples were averaged for each measurement. The resulting total time to identify the orbit originating from one corrector was about $2 \mathrm{~min}$, leading to about $4 \mathrm{~h}$ for identifying 60 correctors, for nominal and dispersive optics, in both planes, over $500 \mathrm{~m}$ of the linac. About $75 \%$ of the total identification time comes from waiting for corrector set point change to stabilize, about $25 \%$ comes from the BPM sampling (100 samples at $30 \mathrm{~Hz}+$ functions overhead). Although the identification is relatively time consuming, once the linac model has been identified one correction iteration takes only $2 \mathrm{~min}$ to perform since the calculation of the correction takes negligible computational time and all corrector set-points can be changed simultaneously. New scripts, based on the SLAC AIDA interface to the EPICS control system [18,19], were developed for the experiment allowing the system identification and the global correction to be applied in a fully automatic manner. A "flight simulator" version of the experiment was created, in which we interfaced the simulation with the experimental scripts that we developed, in order to test all the online scripts on a computer before using them on the real machine.

To identify the response matrix for the dispersive test beam, required for the dispersion correction, we changed the phase of a single klystron upstream the section of interest, from 0 to $90 \mathrm{deg}$. The selection of the klystron and the phase change was based on simulation, and calculated to create an energy difference of the test beam with respect to the main beam of $156 \mathrm{MeV}$ (about $0.78 \%$ relative energy difference at the end of the linac). No change of the quadrupole magnet strength was applied, thus avoiding potential issues with dependence of the magnetic centers of the quadrupoles on the magnetic fields.

\section{RESULTS}

First, system identification for the selected $500 \mathrm{~m}$ of the linac was performed. Figure 6 shows the orbit response matrix, $\mathbf{R}$, as measured by estimating the orbit difference from alternate-sign correctors kicks and then applying the system identification algorithm to construct the response matrix. We then applied a global orbit correction to verify 




FIG. 6. The orbit response matrix, $\mathbf{R}$, as measured by estimating the orbit difference from alternate signs correctors kicks and applying the system identification algorithm.

that the system identification has been successful. The orbit correction was performed by artificially creating an orbit bump with respect to the golden orbit, and automatically steering back to the target orbit (the golden orbit). Figure 7 shows the results of the global orbit correction. The correction minimizes the rms difference of the current orbit (averaged over 100 BPM samples) to the target orbit. The residual difference, of the order of $100 \mu \mathrm{m}$, between the corrected orbit was mainly due to machine drift.

The global dispersion correction algorithm was then applied, as described by Eq. (4). The weighting of the difference orbit with respect to the nominal orbit was set to $\omega=10$, which gave the optimal dispersion reduction after trying a range of weights. This is in good correspondence

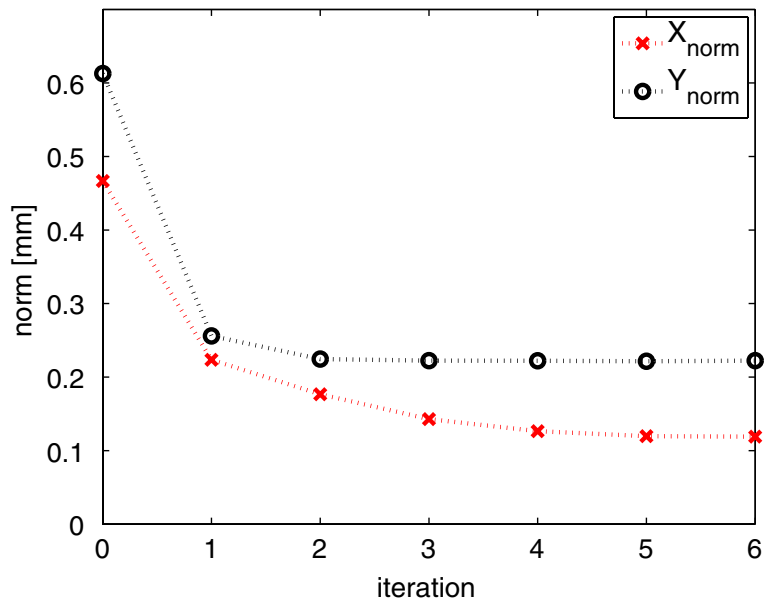

FIG. 7. The rms difference of the current orbit (averaged over 100 BPM samples) with respect to the target orbit (taken here as the golden orbit). This demonstrates that the global orbit correction algorithms successfully reduces the orbit difference, and that the orbit converges in a robust way to a minimum value. In particular, this demonstrates that the machine system identification has been successful. Iteration zero is before the correction. with the theoretical calculation of the weighting, $\omega_{\text {theory }}=14$, calculated from Eq. (3), and with the result of the simulation, as visible in Fig. 5. To stabilize the solution when inverting the system matrix given by Eq. (4), $70 \%$ of the singular values were ignored corresponding to the least significant correction modes. The correction gain used was 0.75 . The results are summarized in Fig. 8 (top). The horizontal dispersion is corrected by a factor 3 and the vertical dispersion by a factor 4 . Note that the rms orbit is not necessarily improved as the dispersion is reduced, as is expected. During the dispersion correction, the tails observed on the transverse profile monitor at the end of the FACET linac were being reduced for each iteration, as shown in Fig. 8 (bottom). The dispersion correction results were reproducible and gave robust convergence, once good parameters for the gain, the weight and the singular value cut were established. Furthermore, the response matrices identified the first day were also shown to give a robust correction in the two days following the system identification, i.e., the FACET machine was stable enough over this period of time to not have to reidentify the machine.

Finally, a dedicated experiment was performed to measure the effect of the dispersion correction on the linac emittance. For this proof-of-principle experiment we artificially spoiled the emittance in the linac by randomly exciting the correctors upstream of the section of interest; this induced an orbit difference between nominal and test beam of $1 \mathrm{~mm}$, which generated a significant emittance growth. Then, the dispersion-free correction was applied and for each iteration the emittance was measured in the first emittance measurement station after the section where the correction was applied, located just after the FACET mid-linac compressor chicane. This measurement station uses four wire scanners. The wire scanners are the same as were designed for the SLAC Linear Collider [20]. Each wire scanner measures the beam profile at a different $z$ location. Based on asymmetric Gaussian fits, the emittance can be estimated, together with the beam Courant-Snyder parameters [21]. The results, shown in Fig. 9, demonstrate that the global dispersion correction successfully reduces the measured emittance. In particular, the vertical emittance is reduced by a factor 30 . Note that in the vertical plane the emittance is reduced simultaneously with the vertical dispersion, while the norm of the vertical absolute orbit is practically constant after the first iteration. This shows that the reduction in dispersion is the cause of the reduction of the emittance. The residual emittances after the corrections were of the order of $10 \mu \mathrm{m}$ in the vertical plane and $40 \mu \mathrm{m}$ in the horizontal plane. Each iteration takes only about $2 \mathrm{~min}$ to perform on $500 \mathrm{~m}$ of the linac. In principle, the correction time per iteration would be of the same order on a larger machine (for example ILC or CLIC) assuming a good model has been established, and assuming all BPMs can be read simultaneously and all correctors can be changed simultaneously. 


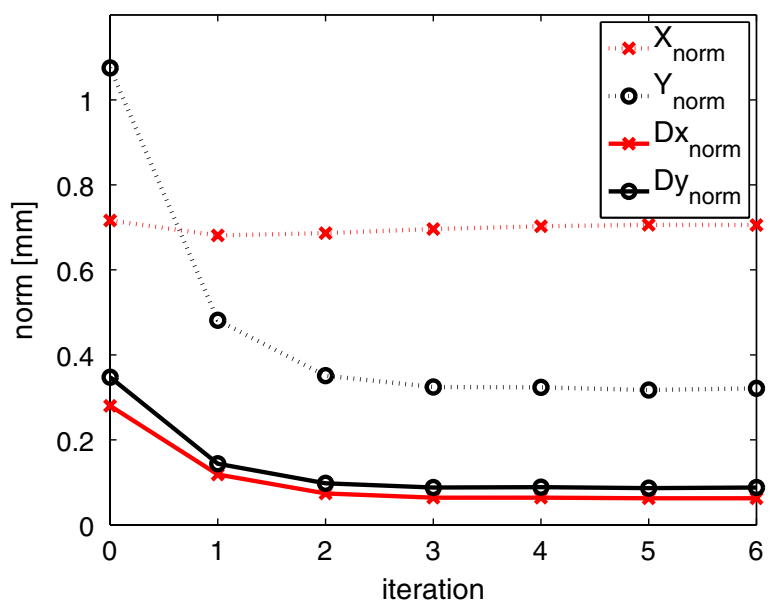

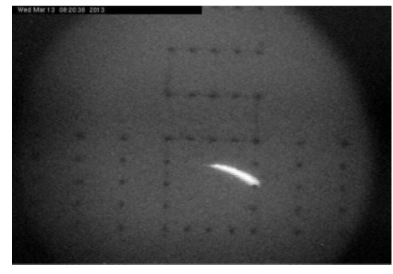

iteration 0

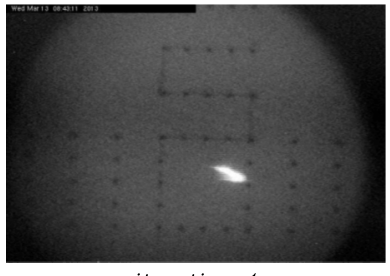

iteration 1

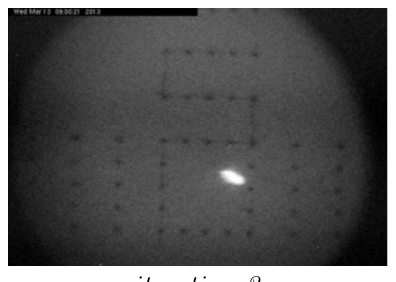

iteration 3

FIG. 8. (top) The rms difference of the current orbit with respect to the target orbit (dotted lines), and the rms value of the difference orbits (full lines). This demonstrates that the algorithm successfully corrects dispersion, and that the dispersion converges in a robust way to a minimum value. The horizontal dispersion is corrected by a factor 3 and the vertical by a factor 4 . Note that the absolute orbit is not necessarily improved as the dispersion is reduced, as is expected. (bottom) Phosphorous beam profile monitor measurements at the end of the FACET linac, before the dispersion correction, after one iteration step and after three iteration steps. Iteration zero is before the correction.

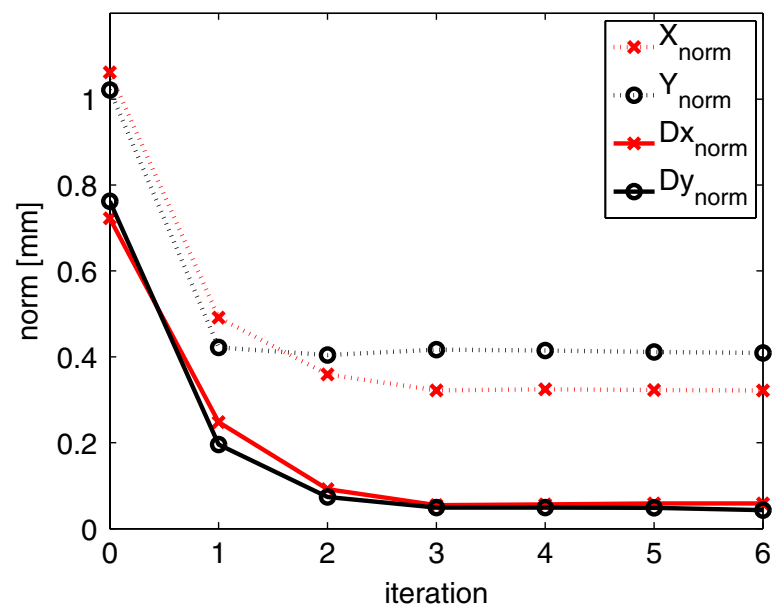

(a)

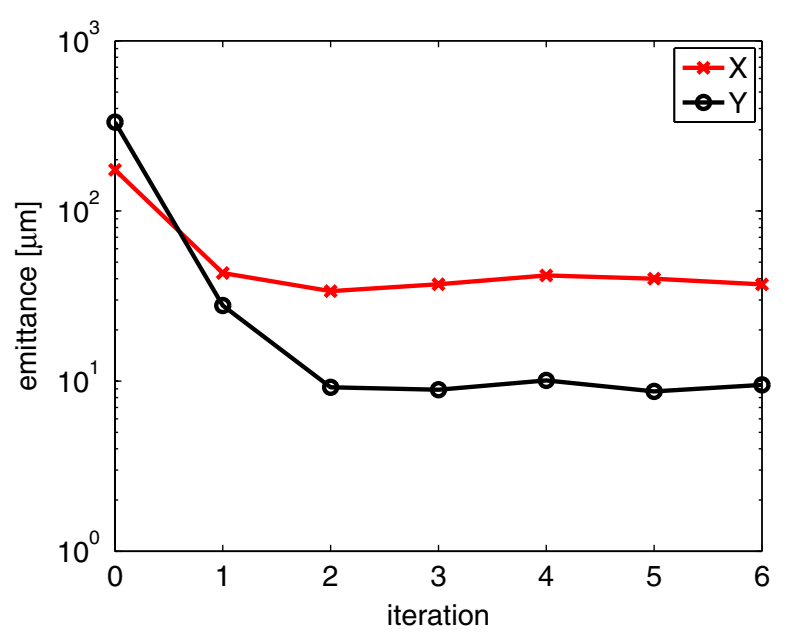

(b)

FIG. 9. (a) The rms difference of the current orbit with respect to the golden orbit (dotted lines), and the rms value of the difference orbits (full lines), over $500 \mathrm{~m}$ in the first half of the FACET linac, for each iteration of the correction. (b) The measured emittance just after the corrected section, as measured for each iteration of the correction. We observe that the global correction successfully reduced the measured emittance. Note that in the vertical plane the emittance is reduced in concert with the vertical dispersion, while the norm of the vertical absolute orbit is practically constant after the first iteration. This shows that the reduction in dispersion is the cause of the reduction of the emittance. Each iteration takes about $2 \mathrm{~min}$ to perform on $500 \mathrm{~m}$ of the linac. Iteration zero is before the correction. 
As shown by the simulation studies reported in Fig. 5, it is probable that the transverse wake fields in offset accelerating structures dominate the residual emittance growth. For the FACET linac we are currently studying a variation of dispersion-free steering using a differentcharge test beam, a "wake-free steering," that may be able to further reduce the emittance. For CLIC or ILC, however, an energy-different test beam, as used in this experiment, is what is foreseen to be used; therefore we consider the demonstration of this technique on the FACET linac as an important milestone in the linear collider studies.

\section{CONCLUSIONS AND OUTLOOKS}

We have demonstrated the proof of principle of a global dispersion-free correction algorithm on $500 \mathrm{~m}$ of the FACET linac. The correction is based on a model automatically identified via a series of measurements, where no a priori information about the machine is required. The dispersion-free correction robustly converges to a solution where the difference orbit of a nominal and a dispersive orbit is minimized. An artificially introduced emittance increase could be reduced by a factor 30 by the DFS procedure, proving that correcting the dispersion effectively improves the emittance. The system identification and correction proposed here was performed in a deterministic, robust, reproducible, and automatic matter, as opposed to an empirical and laborious search for a golden orbit. The success of a global dispersion-free correction is a significant step forward toward the validation of emittance preservation techniques for future linear colliders.

Although dispersive emittance dilution was efficiently corrected by DFS, the FACET linac suffers from significant transverse wake fields, as confirmed by simulations. To achieve further emittance reduction, dispersion-free steering could possibly be improved by measuring the impact of the wakes using a test beam of different charge. We are currently studying a novel scheme for a practical implementation of such a wake-free steering at FACET.

\section{ACKNOWLEDGMENTS}

We are very grateful for the excellent support of the FACET staff and in particular the FACET user manager,
Christine Clarke, over the course of this experiment. We also acknowledge the warm support provided by JeanPierre Delahaye in the early stages of this experiment. This work is supported by the Research Council of Norway.

[1] CLIC Conceptual Design Report, 2012, http://cds.cern.ch/ record/1500095.

[2] ILC Technical Design Report, 2013, http://www .linearcollider.org/ILC/TDR.

[3] T. O. Raubenheimer, Phys. Rev. ST Accel. Beams 3, 121002 (2000).

[4] T. Raubenheimer and R. Ruth, Nucl. Instrum. Methods Phys. Res., Sect. A 302, 191 (1991).

[5] D. Schulte, "Beam-based Alignment in the New CLIC Main Linac" (to be published).

[6] P. Eliasson and D. Schulte, Phys. Rev. ST Accel. Beams 11, 011002 (2008).

[7] E. Adli and D. Schulte, in Proceedings of the 11th European Particle Accelerator Conference, Genoa, 2008 (EPS-AG, Genoa, Italy, 2008), p. 547.

[8] A. W. Chao, Physics of Collective Beam Instabilities in High Energy Accelerators (Wiley, New York, 1993).

[9] J. Pfingstner and A. Latina, CERN Report No. CERNOPEN-2012-020, CLIC-Note-947, 2012.

[10] T. Raubenheimer and P. Tenenbaum, Report No. SLACTN-03-071, 2004.

[11] R. Assmann et al., Report No. SLAC-PUB-10485, 1995.

[12] R. Assmann, P. Raimondi, G. Roy, and J. Wenninger, Phys. Rev. ST Accel. Beams 3, 121001 (2000).

[13] F. J. Decker et al., in Proceedings of the 6th European Particle Accelerator Conference, Stockholm, 1998 (IOP, London, 1998).

[14] FACET User Facility web page, http://facet.slac.stanford .edu.

[15] The tracking code PLACET, https://savannah.cern .ch/projects/placet.

[16] K. J. Aström and B. Wittenmark, Adaptive Control (Dover, New York, 2008).

[17] L. Ljung and S. Gunnardsson, Automatica 26, 7 (1990).

[18] Accelerator Integrated Data Access web page, http://www .slac.stanford.edu/grp/cd/soft/aida.

[19] Experimental Physics and Industrial Control System web page, http://www.aps.anl.gov/epics.

[20] M. Ross et al., Report No. SLAC-PUB-5556, 1991.

[21] M. Ross et al., Report No. SLAC-PUB-4278, 1987. 